\title{
Upregulation of seladin-1 and nestin expression in bone marrow mesenchymal stem cell transplantation via the ERK1/2 and PI3K/Akt signaling pathways in an Alzheimer's disease model
}

\author{
SHI YU ${ }^{1}, \mathrm{YUE} \mathrm{HEI}^{2}$ and WEIPING LIU ${ }^{3}$ \\ ${ }^{1}$ Department of Neurosurgery, No. 303 Hospital of Chinese People's Liberation Army, \\ Nanning, Guangxi 530021; ${ }^{2}$ Department of Neurosurgery, The Fourth Military Medical University, Xi'an, Shaanxi 710000; \\ ${ }^{3}$ Department of Neurosurgery, Xijing Hospital, The Fourth Military Medical University, Xi'an, Shaanxi 710032, P.R. China
}

Received August 14, 2017; Accepted November 21, 2017

DOI: $10.3892 / \mathrm{ol} .2017 .7543$

\begin{abstract}
The aim of the present study was to determine the roles of bone marrow mesenchymal stem cell (BM-MSC) transplantation in a model of Alzheimer's disease (AD) and determine the underlying mechanism. The expression of selective Alzheimer's disease indicator-1 (Seladin-1) and nestin was detected using reverse transcription-quantitative polymerase chain reaction and western blot analysis. The phosphoinositide 3-kinase (PI3K) and extracellular signal-regulated kinase (ERK)1/2 inhibitors, LY294002 and PD98059, were employed to evaluate the molecular mechanism. The results indicated that the mRNA and protein expression of Seladin-1 and nestin was lower in the AD group when compared with the control group. BM-MSC transplantation reversed this decrease in expression, potentially by increasing the protein expression of phosphorylated (p)-protein kinase B (Akt) and p-ERK1/2. In addition, LY294002 (the PI3K inhibitor) and/or PD98059 (the ERK1/2 inhibitor) blocked the enhancement of BM-MSC transplantation on the expression of Seladin-1 and nestin in the hippocampus. These results indicated that BM-MSC transplantation enhanced Seladin-1 and nestin expression potentially via a mechanism associated with the activation of the PI3K/Akt and ERK1/2 signaling pathways. The present study offers preliminary evidence that treatment with BM-MSCs may represent a potential therapeutic approach against brain lesions in AD.
\end{abstract}

Correspondence to: Dr Weiping Liu, Department of Neurosurgery, Xijing Hospital, The Fourth Military Medical University, 127 Changle West Road, Xi'an, Shaanxi 710032, P.R. China

E-mail: 1wp145@yeah.net

Key words: Alzheimer's disease, bone marrow mesenchymal stem cell, extracellular signal-regulated kinases 1/2, phosphoinositide 3-kinase/protein kinase $\mathrm{B}$, rats

\section{Introduction}

Alzheimer's disease (AD) is a degenerative disorder characterized histopathologically by amyloid $\beta(A \beta)$ aggregations, neurofibrillary tangles and neuronal impairment finally leading to progressive loss of recent and episodic memory and other cognitive functions (1). Compared with males, females are more susceptible to suffer from AD (2). At present, therapeutic method for treating AD mainly include increasing the concentration of acetylcholine and other neurotransmitters such as glutamate, while also combining other pharmacological and non-pharmacological strategies for treatment of the AD associated comorbidities to alleviate symptom burden and improve life quality, but still have no practical significance for the progression of $\mathrm{AD}(3)$.

Recent studies have shown that mesenchymal stem cells (MSCs) as a promising therapeutic strategy for AD therapy $(4,5)$. MSCs are multi-potent progenitor cells with ability to differentiate into multiple lineages that are mainly derived from the three sources including bone marrow, adipose tissue, and the umbilical cord. Bone marrow MSCs (BM-MSCs) have properties of self-renewal, rapid proliferation, immunosuppression, low immunogenicity, pluripotency and tissue regeneration and repair ability, which can also increase the number of positive cells for choline acetyltransferase and survivin expression $(4,6)$. Furthermore, human MSCs were able to improve memory by reducing the level of $A \beta$ in the hippocampus and $A \beta$ deposition through the activation of M2-like microglia and modulation neuroinflammation in an A $\beta \mathrm{PP} / \mathrm{PS} 1$ transgenic $\mathrm{AD}$ mouse model (7). Moreover, human MSCs enhanced A $\beta$ immunoreactivity and autophagosome induction, decreased intracellular $\mathrm{A} \beta$ levels, promote $\mathrm{A} \beta$ clearance in $\mathrm{AD}$ models, which may lead to increased neuronal survival against $A \beta$ toxicity (8). Recent work had confirmed that the potential therapeutic role of BMMSCs against AD through their anti-apoptotic, neurogenic and immunomodulatory properties in the experimental AD model, however, the molecular mechanisms are largely unclear.

Selective Alzheimer's disease indicator-1 (Seladin-1) is a specific AD marker and is an important neuroprotective effector. 
A large number of studies have proven that Seladin-1 is closely linked to neuronal degeneration in $\mathrm{AD}$, which is downregulated in brain regions affected by $\mathrm{AD}(9,10)$. Nestin is usually used as a marker of neural stem and progenitor cells. It is expressed in astrocytes and neurons in AD patient and other neurodegenerative disease (11). In addition, nestin transgenic was used to establish AD animal models, and the number of nestin cells showed statistically significant decrease in the dentate gyrus of the hippocampus within the AD groups when compared with the controls (12). Collectively, these data strongly support the idea that Seladin-1 and nestin play pivotal roles in AD pathogenesis, although the exact molecular mechanisms still need to be further elucidated to facilitate better treatment for AD.

The purpose of the current work was to determine the roles of BM-MSCs transplantation in AD model and explore the underlying molecular mechanism. The expression of Seladin-1 and nestin was detected using quantitative polymerase chain reaction (qPCR) and western blot analysis, respectively. We also used the phosphoinositide 3-kinase (PI3K) inhibitor and extracellular signal-regulated kinase (ERK)1/2 inhibitor (LY294002, PD98059) to explore the molecular mechanism. We sought to test the hypothesis that BM-MSCs transplantation regulates Seladin-1 and nestin expression through a mechanism associated with activating the PI3K/protein kinase B (Akt) and ERK1/2 signaling pathways.

\section{Materials and methods}

Animals and experimental groups. All experiments were performed out accordance with the guidelines of the National Institutes of Health for Care and Use of Laboratory Animals and approved by the Animal Welfare Committee of Tangdu Hospital, Fourth Military Medical University. Sprague-Dawley female rats (body weight 130-150 g) were acquired from the Experimental Animal Center of the Fourth Military Medical University (Xi'an, China). The animals were housed at constant temperature at $20-22^{\circ} \mathrm{C}$ and humidity at $40-60 \%$, with a 12 -h day and dark illumination cycle and free access to food and water. All animals were randomized into six groups ( $\mathrm{n}=8$ per group) as follow: Group 1 , rats as a healthy control group; Group 2, rats as Aluminium chloride induced AD (17 mg/kg b. wt daily for 75 days according to previous report (13); Group 3, induced Alzheimer rats that received BM-MSCs only; Group 4, induced Alzheimer rats that received BM-MSCs and LY294002; Group 5, induced Alzheimer rats that received BM-MSCs and PD98059; and Group 6, induced Alzheimer rats that received BM-MSCs, LY294002 and PD98059 treatment group.

Rats in the control group received a lateral ventricular injection of sterile saline $(1 \mathrm{ml})$. As previously described $(14,15)$, $1 \mu \mathrm{g}$ LY294002 (Selleck Chemicals in China, Shanghai, China) and/or $2 \mu \mathrm{g}$ PD98059 (Selleck Chemicals, Shanghai, China) or phosphate-buffered saline (PBS) $10 \mathrm{~min}$ were administered a lateral ventricular injection in the rats after treatment with aluminum chloride. The volume of $2 \mu \mathrm{l}$ was injected in each rat. A single dose of BM-MSCs (3×106 cells/rat) (16) was slowly injected into rats by the tail vein in $5 \mathrm{~min}$ with a $27 \mathrm{G}$ needle.

Primary culture of BM-MSCs. The femoral bones were harvested from 4 donor male rats, and the medullary cavity was bathed with the Dulbecco's modified Eagle's medium (DMEM; Gibco; Thermo Fisher Scientific, Inc., Waltham, MA, USA). Bone marrow cells were cultured in DMEM low glucose (21885-108; Gibco; Thermo Fisher Scientific, Inc.) with $10 \%$ fetal calf serum (SV30160.03; HyClone; GE Healthcare Life Sciences, Logan, UT, USA), 100 U/ml penicillin and $100 \mu \mathrm{g} / \mathrm{ml}$ streptomycin (15070-063; Gibco; Thermo Fisher Scientific, Inc.) $\left(37^{\circ} \mathrm{C}, 5 \% \mathrm{CO}_{2}\right.$ for $\left.48 \mathrm{~h}\right)$, and this culture medium were changed every 3 days. When the adherent cells reached $70-80 \%$ confluence, the cells were passaged at a ratio of $1: 2$ with $0.25 \%$ trypsin (contained $0.02 \%$ EDTA). Identity was confirmed by flow cytometry for MSC-specific markers (the expression of CD105, CD73 and CD90 was more than $95 \%$, whereas the expression of CD34, CD45, CD31 and CD146 was $2 \%$ or less. The fourth generation was used for transplantations in the experiments.

$R T-P C R$. Total RNA was extracted from hippocampus tissue of female rats using TRIzol (Invitrogen, Thermo Fisher Scientific, Inc.). cDNA was synthesized from $1 \mu \mathrm{g}$ of total RNA from each sample using RevertAidTM First Strand cDNA Synthesis kit (no. K1621; Fermentas, Thermo Fisher Scientific, Inc.). The primers used for Seladin-1 were forward, 5'-GGGTGTTTGTGT GCCTCTTCC-3' and reverse, 5'-GCTCCTTCCACTCCCGTA CC-3'; for nestin were forward, 5'-TGGAGCGGGAGTTAGAGG CT-3', and reverse, 5'-ACCTCTAAGCGACACTCCCGA-3'; and for $\beta$-actin were forward, 5'-CTGTCTGGCGGCACCACCAT-3', and reverse, 5'-GCAACTAAGTCATAGTCCGC-3 (17). RT-PCR was performed in a Bio-Rad IQ ${ }^{\mathrm{TM}}$ system (Bio-Rad Laboratories, Inc., Hercules, CA, USA) using SYBR-Green qPCR analysis. A total of 40 PCR cycles were used for amplification of Seladin-1, nestin, and $\beta$-actin. We used $\Delta \mathrm{Cq}$ method to estimate the relative quantification of Seladin- 1 and nestin mRNA. The $\beta$-actin amplification levels were used as reference to normalize the relative quantification of the target gene.

Western blot analysis. Hippocampus tissue were homogenized and lysed with tissue protein lysis solution. Protein concentrations were determined using BCA kit. Standard samples were fractionated on a 10\% SDS-PAGE gel, and then transferred onto polyvinylidene difluoride (PVDF) membranes and blocked with $5 \%$ bovine serum albumin (BSA) at room temperature for $2 \mathrm{~h}$. Followed by incubation with Seladin-1 (2033S; Santa Cruz Biotechnology, Inc., Dallas, TX, USA), nestin (4760S; Santa Cruz Biotechnology, Inc.), Akt (1085S; Santa Cruz Biotechnology, Inc.), p-Akt (10001S; Santa Cruz Biotechnology, Inc.), ERK1/2 (1240S; Santa Cruz Biotechnology, Inc.), p-ERK1/2 (1150S; Santa Cruz Biotechnology, Inc.), and $\beta$-actin ((13E5) Rabbit mAb (Alexa Fluor ${ }^{\circledR} 594$ Conjugate) $9470 S$; Santa Cruz Biotechnology, Inc.) (all antibody were 1:1,000 diluted) overnight at $4^{\circ} \mathrm{C}$ followed by incubation with secondary antibody (1:5,000 diluted; Cell Signaling Technology, Inc., Danvers, MA, USA). Signal was visualized with enhanced chemiluminescence (ECL) reagent in the Tanon 4500 imaging system and the band intensities were quantified using ImageJ software. Normalization was based on the protein level of $\beta$-actin.

Statistical analysis. All data are presented as the mean \pm standard deviation. Data were tested for normality 
A
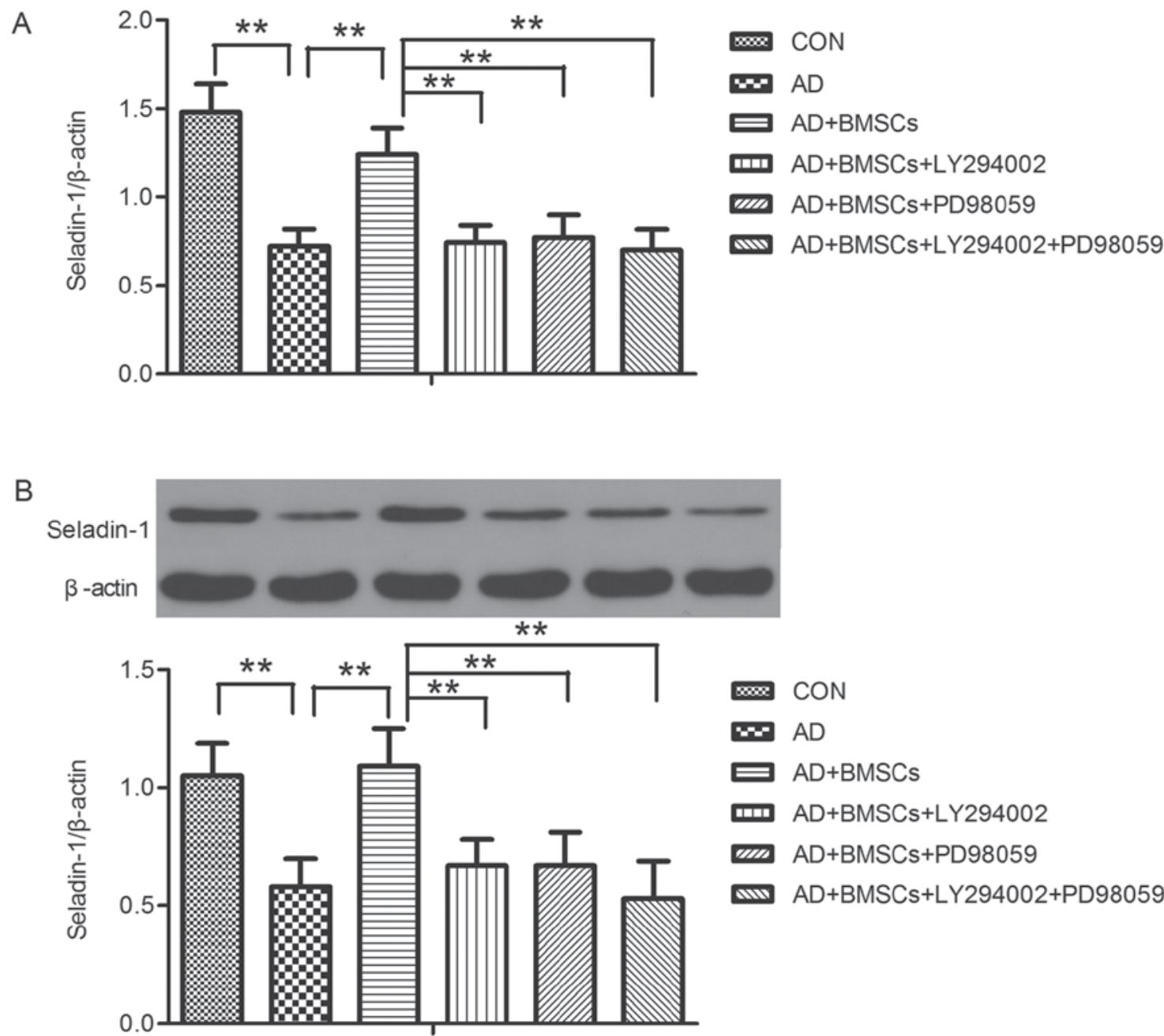

Figure 1. Effect of (A) varying treatments on brain seladin-1 gene expression determined by (B) reverse transcription-polymerase chain reaction and protein expression evaluated by western blot analysis in the AD model. Data are presented as the mean \pm standard deviation ( $\mathrm{n}=8 / \mathrm{group}$ ). ${ }^{* *} \mathrm{P}<0.001$, as indicated. $\mathrm{AD}$, Alzheimer's disease; BMSCs, bone marrow mesenchymal stem cells; CON, control.

used Kolmogorov-Smirnov test. The equality of variances between the groups was assessed used Levene's test. For statistical analysis, significant differences were analyzed using a one-way ANOVA analysis followed by the Tukey post-hoc comparisons (SPSS 17.0 software; SPSS, Inc., Chicago, IL, USA). P<0.05 was considered to indicate a statistically significant difference.

\section{Results}

Molecular study of Seladin-1 gene expression and protein expressions in AD model. The results of RT-PCR showed that Seladin-1 gene level showed significant difference between groups $[\mathrm{F}(5,42)=55.046, \mathrm{P}<0.001]$ (Fig. 1A). Tukey post hoc test revealed that Seladin-1 mRNA level was lower in the AD group compared to that in the control group $(\mathrm{P}<0.001$, AD vs. CON group). After BM-MSCs transplantation, the Seladin-1 mRNA level was significantly increased ( $\mathrm{P}<0.001, \mathrm{BM}-\mathrm{MSCs}$ vs. AD group). When treated with a PI3K and p-ERK1/2 inhibitor, LY294002, PD98059 or combination the two inhibitors, Seladin-1 mRNA level was significantly decreased (all P<0.001, LY294002, PD98059 or LY294002 + PD98059 vs. BM-MSCs group, respectively).
Furthermore, we used western blot analysis to detect the change of Seladin-1 protein expression, and the results showed as that of Seladin-1 mRNA. There were significant differences of Seladin-1 protein expression between groups $[\mathrm{F}=24.508(5,42), \mathrm{P}<0.001]$ (Fig. 1B). After Tukey post-hoc test, the AD group showed a significant decrease in brain Seladin-1 protein expression in comparison with the control group ( $\mathrm{P}<0.001$, AD vs. CON group), BM-MSCs transplantation significantly increased Seladin-1 protein expression in comparison with the AD group $(\mathrm{P}<0.001, \mathrm{BM}-\mathrm{MSC}$ s v. AD group), but treatment of the AD group with LY294002, PD98059 or LY294002+ PD98059 significantly decreased brain Seladin-1 protein expression respectively in comparison with the BM-MSCs group (all P<0.001, LY294002, PD98059 or LY294002 + PD98059 vs. BM-MSCs group, respectively).

Molecular study of nestin gene and protein expressions in AD model. Fig. 2 represents the effect of treatment with LY294002, PD98059, or BM-MSCs on brain nestin gene and protein expression in AD model. There was significant difference on the nestin gene level between groups $[F(5,42)=35.852, P<0.001]$. Tukey post hoc test showed that 

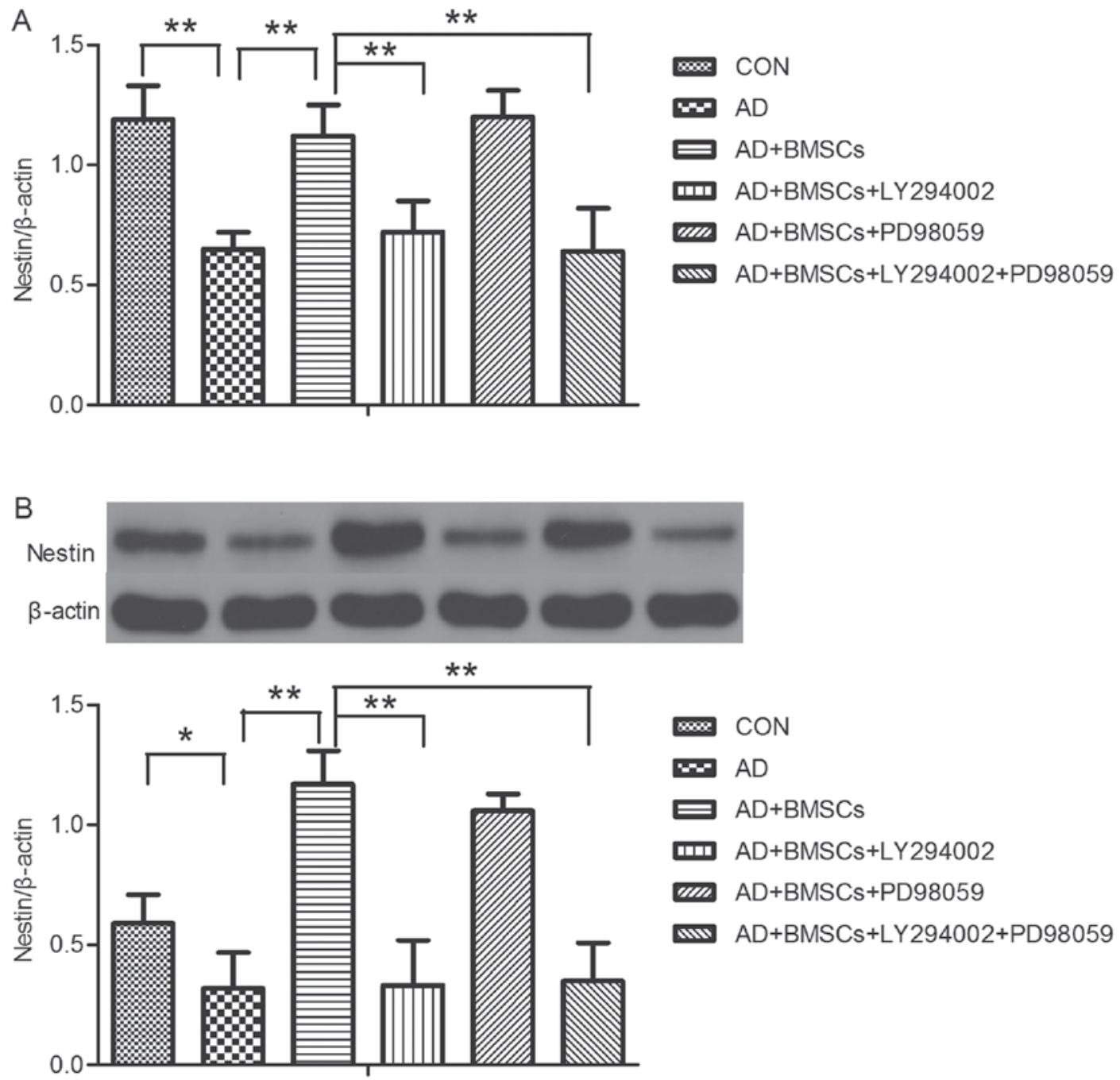

Figure 2. Effect of (A) varying treatments on brain Nestin gene expression determined by (B) reverse transcription-polymerase chain reaction and protein expression evaluated by western blot analysis in the AD model. Data are presented as the mean \pm standard deviation ( $\mathrm{n}=8 /$ group). ${ }^{*} \mathrm{P}<0.05$ and ${ }^{* * *} \mathrm{P}<0.001$, as indicated. AD, Alzheimer's disease; BMSCs, bone marrow mesenchymal stem cells; CON, control.

nestin mRNA level was lower in the AD group compared to that in the control group $(\mathrm{P}<0.001, \mathrm{AD}$ vs. $\mathrm{CON}$ group). After BM-MSCs transplantation, the nestin mRNA level was significantly increased $(\mathrm{P}<0.001$, BM-MSCs vs. AD group). When treated with a PI3K inhibitor, LY294002, nestin mRNA level was significantly decreased (all P<0.001, LY294002, or LY294002 + PD98059 group vs. BM-MSCs, respectively), while there was no effect of PD98059 on the nestin mRNA level ( $\mathrm{P}>0.05$, PD98059 vs. BM-MSCs group). The expression of nestin was analyzed using western blot analysis, and the result showed that there was significant difference of nestin protein expression between groups $[\mathrm{F}=57.439(5,42)$, $\mathrm{P}<0.001]$. After Tukey post-hoc test, the AD group showed a significant decrease in the nestin protein expression in comparison with the control group ( $\mathrm{P}=0.007, \mathrm{AD}$ vs. $\mathrm{CON}$ group), BM-MSCs transplantation significantly increased nestin protein expression in comparison with the $\mathrm{AD}$ group ( $\mathrm{P}<0.001, \mathrm{BM}-\mathrm{MSCs}$ vs. AD group), treatment of the AD group with LY294002 or LY294002 + PD98059 significantly decreased in the nestin protein expression respectively in comparison with the BM-MSCs group (all $\mathrm{P}<0.001$, LY294002 or LY294002 + PD98059 vs. BM-MSCs group, respectively), but there was no effect of PD98059 on the nestin protein expression ( $\mathrm{P}>0.05$, PD98059 vs. BM-MSCs group).

Activation of PI3K/Akt pathway after BM-MSCs transplantation in $A D$ model. As shown in Fig. 3, there was significant difference on the Akt and p-Akt protein expression between groups $[\mathrm{F}(5,42)=2.949, \mathrm{P}=2.949 ; \mathrm{F}(5,42)=26.237, \mathrm{P}<0.001]$. Tukey post-hoc test showed that Akt and p-Akt protein expression was lower in the $\mathrm{AD}$ group in comparison with the control group ( $\mathrm{P}=0.004, \mathrm{P}<0.001$ respectively, $\mathrm{AD}$ vs. $\mathrm{CON}$ group). BM-MSCs transplantation significantly enhanced p-Akt protein expression in comparison with the AD group $(\mathrm{P}<0.001$, BM-MSCs vs. AD group). PD98059 or/and LY294002 treatment dramatically inhibited p-Akt expression (all $\mathrm{P}<0.001$, LY294002, PD98059 or LY294002 + PD98059 vs. BM-MSCs group).

Activation of ERK1/2 pathway after BM-MSCs transplantation in $A D$ model. Fig. 4 showed that there was significant difference on the p-ERK $1 / 2$ protein expression between groups $[\mathrm{F}(5,42)=18.812, \mathrm{P}<0.001]$. Tukey post-hoc test 

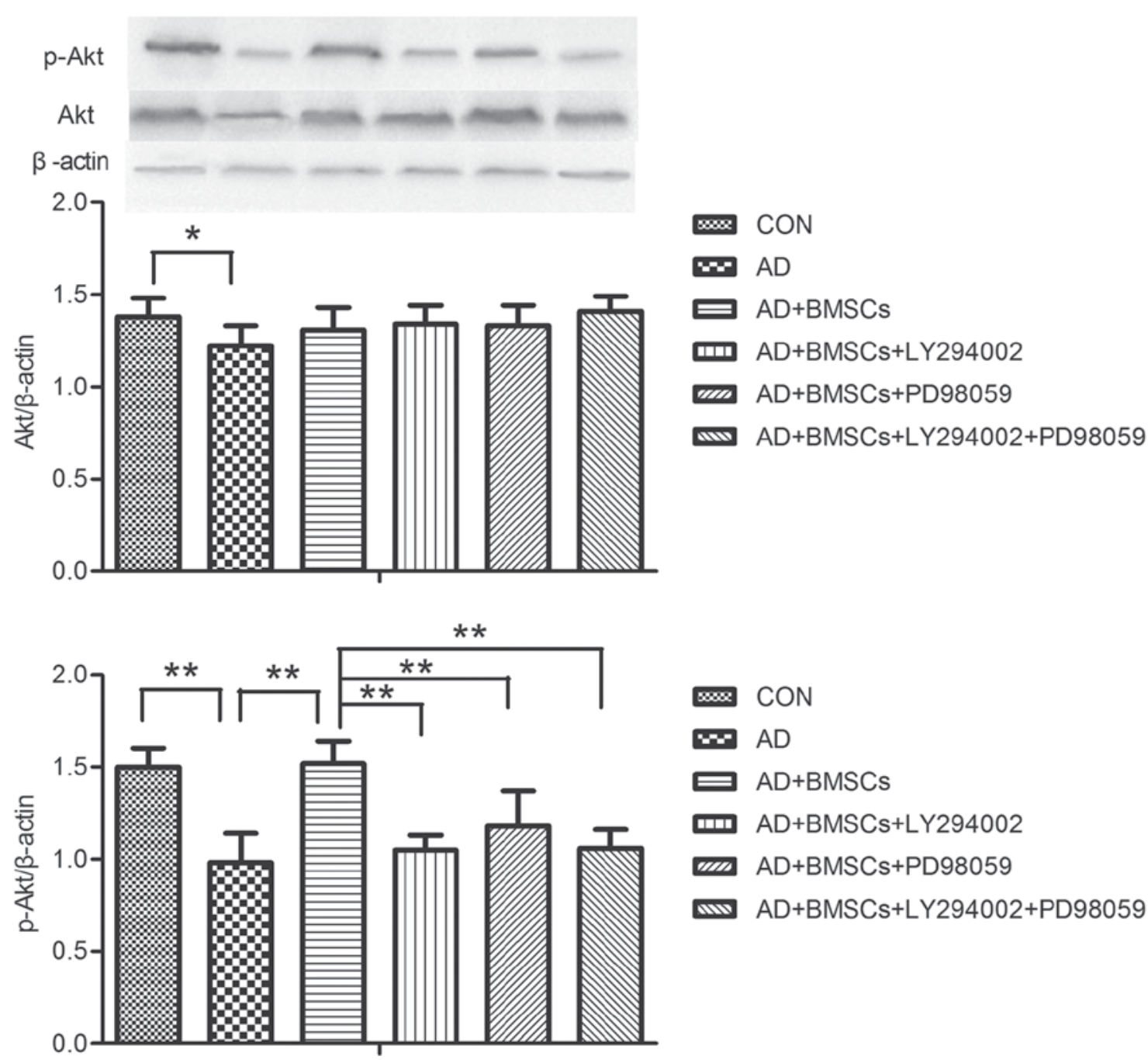

Figure 3. Activation of the phosphoinositide 3-kinase/Akt signaling pathway following BM-MSCs transplantation. Western blot analysis evaluated the protein levels of Akt and p-Akt in the hippocampus tissue following the different treatments. Data are presented as the mean \pm standard deviation ( $\mathrm{n}=8 / \mathrm{group}$ ). $\mathrm{P}<0.05$ and ${ }^{* *} \mathrm{P}<0.001$, as indicated. AD, Alzheimer's disease; BMSCs, bone marrow mesenchymal stem cells; CON, control; Akt, protein kinase B; p-, phosphorylated.

showed that $\mathrm{p}$-ERK1/2 protein expression was lower in the AD group in comparison with the control group $(\mathrm{P}<0.001$, AD vs. CON group). BM-MSCs transplantation significantly increased $\mathrm{p}$-ERK $1 / 2$ protein expression in comparison with the AD group ( $\mathrm{P}<0.001, \mathrm{BM}-\mathrm{MSC}$ vs. AD group). LY294002 treatment significantly attenuated p-ERK1/2 expression ( $\mathrm{P}<0.001$, LY294002 vs. BM-MSCs group). There was no significant difference of p-ERK1/2 expression between PD98059 or LY294002 + PD98059 and BM-MSCs group (all P>005, PD98059 or LY294002 + PD98059 vs. BM-MSCs group).

\section{Discussion}

Aluminum chloride was widely used as an inductor for AD model $(18,19)$. Aluminum exposure significantly lowered the acetylcholinesterase activity in the crude synaptosomal fraction of cortex, hippocampus, thalamus and nucleus caudatus in Mongolian gerbils comparing to the control group (20). The in vitro experiment also indicated that aluminum chloride was potent enough to significantly inhibit acetylcholinesterase (21). When the cultured human lymphocytes were treated with 5,
10,15 and $25 \mu \mathrm{M}$ concentrations of aluminum, all phases of the cell cycle resulted in polyploidy and endoreduplication and DNA damage (22). These data indicate that $\mathrm{AlCl} 3$ is a valid inductor for AD model.

In the present study we demonstrated that the expression of Seladin-1 gene and protein was decreased in the AlCl3-induced $\mathrm{AD}$ model compared with that in the control rats. After a single dose of BM-MSCs $\left(3 \times 10^{6}\right.$ cells/rat $)$ into the hippocampus, the expression of Seladin-1 gene and protein was found to be enhanced, supporting the data of Abdel Aziza et al (23) who reported that BM-MSCs exert beneficial effects in AD rats possibly through decreasing the brain cholesterol level and increasing Seladin-1 gene expression. Moreover, the results of the therapeutic potential of comparing BM-MSCs with 2 conventional therapies (Rivastigmine and Cerebrolysin) of AD also indicated that BM-MSCs exerted the potential therapeutic role via enhancing the Seladin-1 and nestin gene expression (19). Nestin is an intermediate filament protein expressed in the neural progenitor cells and it's also a marker of proliferating and migrating cells. The decreased expression in nestin gene and protein in the AD rats in the present study was detected, but BM-MSCs transplantation significantly reversed this reduction, 

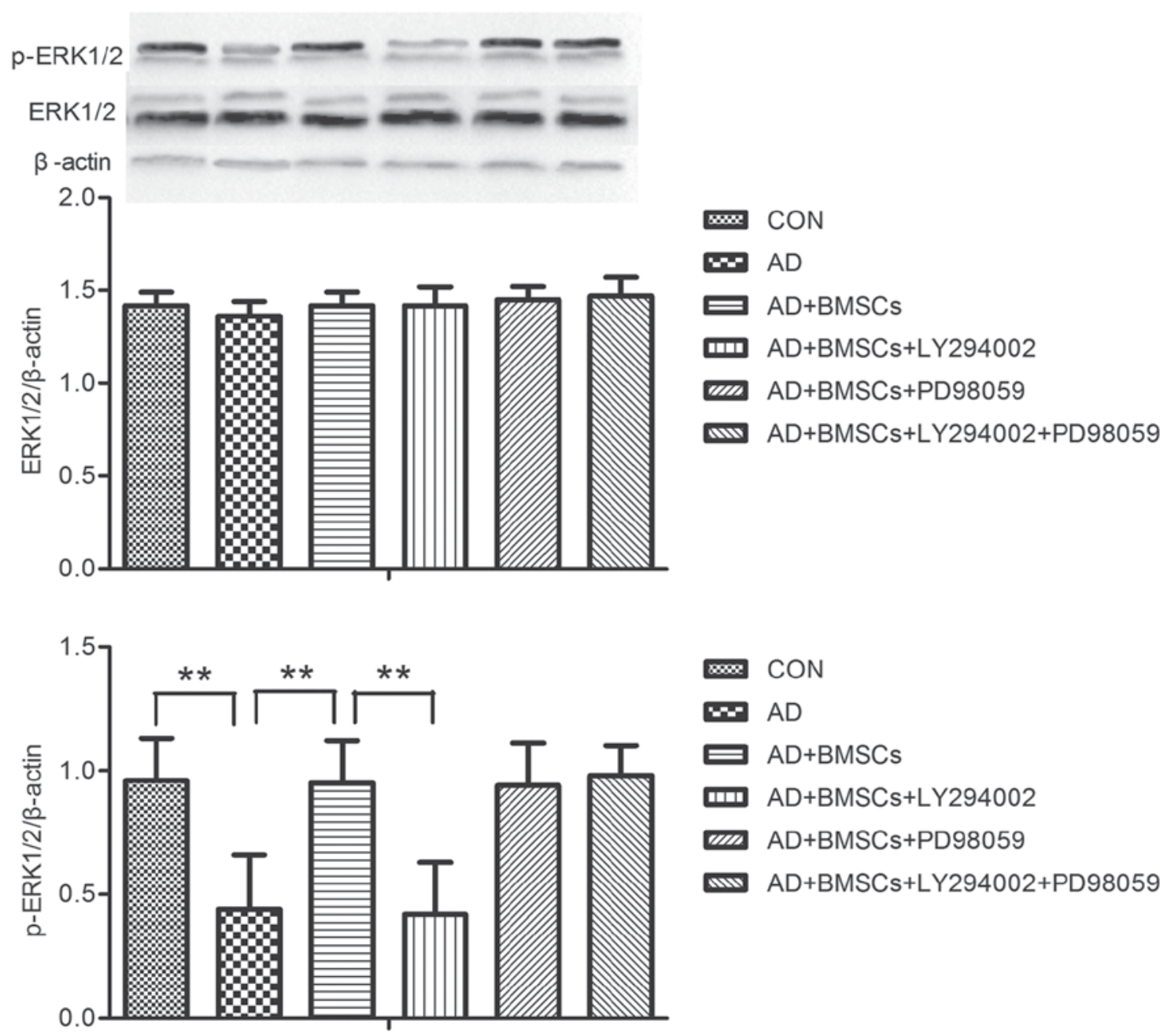

Figure 4. Activation of the ERK1/2 signaling pathway following BM-MSCs transplantation. Western blot analysis evaluated the protein levels of ERK1/2 and p-ERK1/2 in the hippocampus tissue following the different treatments. Data are presented as the mean \pm standard deviation ( $=8 /$ group). ${ }^{* *} \mathrm{P}<0.001$, as indicated. AD, Alzheimer's disease; BMSCs, bone marrow mesenchymal stem cells; CON, control; ERK, extracellular signal-regulated kinase; p-, phosphorylated.

which showed that BM-MSCs exerted the potential therapeutic role for AD possibly via enhancing the expression of Seladin-1 and nestin gene or protein. To further elucidate the underlying molecular mechanisms, we employed two indictors (LY294002, PD98059) of PI3K and p-ERK1/2 signaling pathway and found that treatment with LY294002 effectively blocked the therapeutic effect of BM-MSCs as indicated from the decreasing expression levels of Seladin-1, nestin, p-Akt and p-ERK1/2. Treatment with PD98059 decreased the expression of Seladin-1, p-Akt, but no action on the expression of nestin and p-ERK1/2. Treatment with LY294002 and PD98059 also decreased the expression of Seladin-1, nestin, p-Akt, but no impact on the expression of p-ERK1/2. These observations indicated that PI3K and ERK1/2 signaling pathway were closely linked the mechanism of the therapeutic role of BM-MSCs transplantation for AD.

The aim of the present work is to present a preliminary study of the effect BM-MSCs transplantation on the expression of Seladin-1 and nestin and the underlying molecular mechanisms in Alzheimer model. There are some limitations to our study. First, although aluminum exposure had been confirmed as a valid inductor for constructing AD model, and aluminum exposure rats showed significant increase in escape latency and decrease in the percentage of time in residence in the original platform quadrant in Morris water maze, we should also use some methods such as morris water maze to detect the valid of our present AD model. Second, cells composition in the hippocampus tissue is complex, we detected the whole RNA and protein, but did not used immunohistochemistry and immunofluorescence test to confirm which cell type from of the RNA and protein. Third, due to the design of the experiment is preliminary, the study only offer preliminary evidence that treatment with BM-MSCs may represent the potential therapeutic approach against the brain lesion in AD. Our future direction of work will be proceed based on all above limitations.

In summary, the results from this study indicated that BM-MSCs transplantation enhanced the Seladin-1 and nestin expression may through a mechanism associated with activating the PI3K/Akt and ERK1/2 pathways. Our study offers preliminary evidence that treatment with BM-MSCs may represent the potential therapeutic approach against the neurodegeneration characterized AD.

\section{References}

1. Bekris LM, Yu CE, Bird TD and Tsuang DW: Genetics of Alzheimer disease. J Geriatr Psychiatry Neurol 23: 213-227, 2010. 
2. Podcasy JL and Epperson CN: Considering sex and gender in Alzheimer disease and other dementias. Dialogues Clin Neurosci 18: 437-446, 2016.

3. Jicha GA and Carr SA: Conceptual evolution in Alzheimer's disease: Implications for understanding the clinical phenotype of progressive neurodegenerative disease. J Alzheimers Dis 19: 253-272, 2010

4. Li M, Guo K and Ikehara S: Stem cell treatment for Alzheimer's disease. Int J Mol Sci 15: 19226-19238, 2014.

5. Naaldijk Y, Jäger C, Fabian C, Leovsky C, Blüher A, Rudolph L, Hinze A and Stolzing A: Effect of systemic transplantation of bone marrow-derived mesenchymal stem cells on neuropathology markers in APP/PS1 Alzheimer mice. Neuropathol Appl Neurobiol 43: 299-314, 2017

6. Tirino V, Paino F, D'Aquino R, Desiderio V, De Rosa A and Papaccio G: Methods for the identification, characterization and banking of human DPSCs: Current strategies and perspectives Stem Cell Rev 7: 608-615, 2011.

7. Yang H, Xie Z, Wei L, Yang H, Yang S, Zhu Z, Wang P, Zhao C and Bi J: Human umbilical cord mesenchymal stem cell-derived neuron-like cells rescue memory deficits and reduce amyloid-beta deposition in an AßPP/PS1 transgenic mouse model. Stem Cell Res Ther 4: 76, 2013.

8. Shin JY, Park HJ, Kim HN, Oh SH, Bae JS, Ha HJ and Lee PH: Mesenchymal stem cells enhance autophagy and increase $\beta$-amyloid clearance in Alzheimer disease models. Autophagy 10: 32-44, 2014.

9. Iivonen S, Hiltunen M, Alafuzoff I, Mannermaa A, Kerokoski P, Puoliväli J, Salminen A, Helisalmi S and Soininen H: Seladin-1 transcription is linked to neuronal degeneration in Alzheimer's disease. Neuroscience 113: 301-310, 2002.

10. Benvenuti S, Saccardi R, Luciani P, Urbani S, Deledda C, Cellai I, Francini F, Squecco R, Rosati F, Danza G, et al: Neuronal differentiation of human mesenchymal stem cells: Changes in the expression of the Alzheimer's disease-related gene seladin-1. Exp Cell Res 312: 2592-2604, 2006.

11. Lu G, Kwong WH, Li Q, Wang X, Feng Z and Yew DT: Bcl2, bax and nestin in the brains of patients with neurodegeneration and those of normal aging. J Mol Neurosci 27: 167-174, 2005.

12. Zeng Q, Zheng M, Zhang T and He G: Hippocampal neurogenesis in the APP/PS1/nestin-GFP triple transgenic mouse model of Alzheimer's disease. Neuroscience 314: 64-74, 2016.

13. Krasovskiĭ GN, Vasukovich LY and Chariev OG: Experimental study of biological effects of leads and aluminum following ora administration. Environ Health Perspect 30: 47-51, 1979.

14. Barros DM, Mello e Souza T, de Souza MM, Choi H, DeDavid e Silva T, Lenz G, Medina JH and Izquierdo I: LY294002, an inhibitor of phosphoinositide 3-kinase given into rat hippocampus impairs acquisition, consolidation and retrieval of memory for one-trial step-down inhibitory avoidance. Behav Pharmacol 12: 629-634, 2001.
15. Zamin LL, Dillenburg-Pilla $P$, Argenta-Comiran R, Horn AP, Simão F, Nassif M, Gerhardt D, Frozza RL and Salbego C: Protective effect of resveratrol against oxygen-glucose deprivation in organotypic hippocampal slice cultures: Involvement of PI3-K pathway. Neurobiol Dis 24: 170-182, 2006

16. Zhao DC, Lei JX, Chen R, Yu WH, Zhang XM, Li SN and Xiang P: Bone marrow-derived mesenchymal stem cells protect against experimental liver fibrosis in rats. World $\mathrm{J}$ Gastroenterol 11: 3431-3440, 2005

17. Gaytan F, Barreiro ML, Caminos JE, Chopin LK, Herington AC, Morales C, Pinilla L, Paniagua R, Nistal M, Casanueva FF, et al: Expression of ghrelin and its functional receptor, the type 1a growth hormone secretagogue receptor, in normal human testis and testicular tumors. J Clin Endocrinol Metab 89: 400-409, 2004.

18. Zhang QL, Jia L, Jiao X, Guo WL, Ji JW, Yang HL and Niu Q: APP/PS1 transgenic mice treated with aluminum: An update of Alzheimer's disease model. Int J Immunopathol Pharmacol 25: 49-58, 2012.

19. Salem AM, Ahmed HH, Atta HM, Ghazy MA and Aglan HA: Potential of bone marrow mesenchymal stem cells in management of Alzheimer's disease in female rats. Cell Biol Int 38: 1367-1383, 2014

20. Vučetić-Arsić S, Radonjić NV, Jovanović M, Selaković V, Nikolić T, Velimirović M, Stojković T, Milovanović A, Milovanović J and Petronijević ND: Oxidative stress precedes mitochondrial dysfunction in gerbil brain after aluminum ingestion. Environ Toxicol Pharmacol 36: 1242-1252, 2013.

21. Pohanka M: Copper, aluminum, iron and calcium inhibit human acetylcholinesterase in vitro. Environ Toxicol Pharmacol 37: 455-459, 2014.

22. Lima PD, Leite DS, Vasconcellos MC, Cavalcanti BC, Santos RA, Costa-Lotufo LV, Pessoa C, Moraes MO and Burbano RR: Genotoxic effects of aluminum chloride in cultured human lymphocytes treated in different phases of cell cycle. Food Chem Toxicol 45: 1154-1159, 2007.

23. Abdel Aziza MT, Atta HM, Samer H, Ahmed HH, Rashed LA, Sabry D, Abdel Raouf ER and Alkaffas MA: Heme oxygenase effect on mesenchymal stem cells action on experimental Alzheimer's disease. EXCLI J 12: 778-792, 2013. 